\title{
SULLA RIDUZIONE REGIONALE E SUL METODO DELLE DERIVATE SECONDE IN GRAVIMETRIA
}

\section{Antonio Marussi}

1. In alcuni recenti lavori $\left(^{1}\right)$ sono stati illustrati particolari metodi per la interpretazione delle misure di gravità, che tendono a mettere in risalto le cosidette anomalie locali, dovute a distribuzioni di densità localizzate e poco profonde, quali sono per lo più quelle che interessano la prospezione geomineraria; questi metodi si basano sostanzialmente sullo studio delle differenze fra il valore $g_{0}$ della gravità in un punto $P_{o}$, e la media (o le medie) $g_{R}$ dei valori $g$ su uno (o più) cerchi di opportuno raggio $R$ aventi $P_{0}$ come centro, tracciati sulla superficie equipotenziale $\Sigma$ per $P_{0}$.

Si prescinde qui dai procedimenti adottati per ridurre alla superficie equipotenziale $\$ i valori della gravità effettivamente osservati, notando solo che le conclusioni che seguono valgono ovviamente per il campo potenziale della gravità modificato in seguitor alle ipotesi che stanno a fondamento di tali riduzioni. Alcune formule si possono però applicare anche quando la superficie sulla quale si considera la data distribuzione della gravità, è una superficie qualunque, anche ad esempio la superficie fisica del terreno; in tal caso i valori della gravità da considerare sono quelli effettivamente osservati, e le conclusioni si riferiscono al campo reale della gravità.

Tralasciando queste considerazioni, sulle quali non intendiamo insistere qui, i metodi accennati danno luogo precisamente:

a) alla riduzione regionale, mediante la quale ci si propone di mettere in risalto le anomalie locali, togliendo al valore della gravità in ogni punto, la media dei valori di questa lungo una circonferenza di raggio prefissato avente il punto considerato come centro;

b) al procedimento delle derivate seconde, che consiste nel ripetere il calcolo ricordato or ora per una serie di circonferenze aventi il punto considerato come centro, e raggi via via decrescenti. Il limite cui tendono i valori così ottenuti, divisi per il quadrato del raggio della circonferenza rispettiva, si assume allora uguale alla derivata seconda $\frac{d^{2} g}{d v^{2}}$ della gravità nel senso della verticale $v$. 
In questa Nota ci si propone di mostrare come in amberlue questi metodi i risultati possano metlersi in relazione con il secondo para-

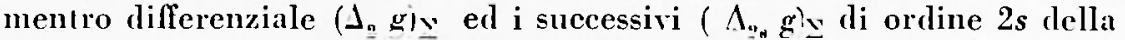
gravita g sulla superficie `̀ considerata, valendosi di alcuni risultati ottenuti ancora nel 1909 dal Pizzctli ( $\left.{ }^{2}\right)$; e di illustrare poi il significato geometrico e meccanico, in relazione alla strutlura locale del campo di gravitì, di tale paramentro.

2. Prima di procedere, ricordiamo perciò i risultati ottenuti dal Pizzelti. Detto $g_{0}$ il valore di una funzione $g$ in un punto $P_{0}$ del piano, $g_{\|}$la media arimetica dei valori che la funzione stessa assume nei punti della circonferenza di ragrgio $R$ c cento $P_{\text {on }}$ si ha

$$
\begin{gathered}
g_{\mathrm{R}}=g_{0}+\frac{R^{2}}{\dot{L}^{2}}\left(\Delta_{2} g\right)_{\Sigma}+\frac{R^{4}}{2^{2} 4^{2}}\left(\Delta_{1} g\right)_{\Sigma}+\ldots \\
\ldots+\frac{R^{2 n-2}}{2^{2} 4^{2} \ldots\left(2_{n}-2\right)^{2}}\left(\Delta_{2 n-2} g\right)_{\triangle}+\frac{R^{2 n}}{2^{2} 4^{2} \ldots(2 n)^{2}}\left(\overline{J_{2 n} g}\right)_{\triangle}
\end{gathered}
$$

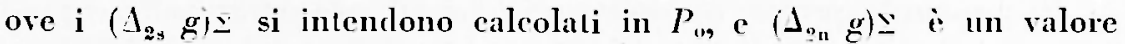
compreso fra il massimo ed il minimo di quelli che $\Lambda_{2 n} g$ assume nell'area circolare considerata.

Tale formula si applica soltanto, come si verle, al caso del piano, o delle superfici sviluppabili; e la sua estensione al caso delle superfici qualsivogliano non $\dot{e}$ immediata; ma invece $\dot{c}$ ancora vera, come il Pizzetti ha dimostralo, anche per le superfici qualsivorliano, la relazione, orvia per il piano,

$$
\lim _{R \rightarrow 0} \frac{4\left(g_{1}-g_{0}\right)}{R^{2}}=\left(\Lambda_{\sigma_{2}} g\right)_{2} .
$$

In questo caso gli enunciati precerlenti si morlificano sostituendo la circonferenza geodetica di raggio $R$ alla circonferenza del piano.

3. Ricordando ora la definizione che è stata data della riduzione regionale, ben si vede come questa possa essere espressa - nell'ipotesi in cui in prima approssimazione la superficie alla quale sono ridotli $i$ valori di g possa confondersi con il piano - con lo sviluppo per le potenze pari di $R$ dato dal Pizzetti, i cui coefficienti sono espressi mediante $i$ secondi parametri differenziali superficiali successivi di $g$; $i$ quali rengono dunque ad assumere un ruolo importantissimo nello studio delle anomalic locali, dovute a distribuzioni di densita situate a piccola profondita; fra questi il sccondo parametro differenziale 
$\left(\Delta_{y} g\right) \Sigma$ ì poi quello particolarmente interessante, poiché esso può essere calcolato agevolmente come il limite dato dal Pizzetli o, più facilmente ancora, per la regola dell'Hospital, come

$$
(\Delta, g)_{\triangle}=4 \lim _{R \rightarrow 0} \frac{d g_{\bar{n}}}{d\left(R^{2}\right)} \text {. }
$$

Calcolati precisamente i valori medi $g_{k}$ su di una serie di circonferenze di raggio decrescente, e riportati i quadrupli dei valori oltenuti come ordinate in un sistema cartesiano in cui l'asse delle ascisse abbia come parametro $R^{2}$, l'angolo che la tangente nell'origine alla curva oftenuta raccordando i punti rappresentativi forma con l'asse delle ascisse, ha come tangente il $\left(\Delta_{:} g\right)$ : cercato.

Altri procedimenti più rapidi, se pure meno precisi, possono venire applicati per il calcolo numerico di detto (\lrcorner$\left._{2} g\right) \mathrm{s}$.

4. Ciò premesso, veniamo a studiare più da vicino il significato meccanico del secondo paramentro differenziale di Beltrani della gravita, su di una superficie Z che, per ragioni di semplicità, ammetteremo essere superficie equipotenziale o di livello del campo; si intende che, se i valori della gravita non fossero stati misurati direttamente su questa, essi dorrebbero immaginarsi ad essa ridotti secondo i consueti procedimenti convenzionali.

Potremo infine definire il seconlo parametro differenziale scrivendo in forma rettoriale [3]

$$
\left(\Delta_{2} g\right)_{\Sigma}=\text { div grad } g_{\Sigma} g
$$

in eui

$$
\operatorname{grad}_{\Sigma} g=\text { grad } g-\operatorname{grad} g \times v, v
$$

: la componente superficiale su $\searrow$ del gradiente di g. Con $v$ si è indicato il versore della normale a \ (diretto verso l'alto), che caratterizza la congruenza rettilinea delle normali; nel mentre con $n$ indicheremo nel seguito il versore delle tangenti alle linee di forza del campo, pure diretto verso l'alto, che caratterizza invece la congruenza curvilinea delle linee di forza. Si ha dunque $n=v$ su $\Sigma$, e poi

$$
\frac{d n}{d P}=\sigma \quad: \quad \frac{d v}{d P}=\sigma_{\Sigma}
$$

$\sigma$ essendo l'omografia di Burali-Forti generalizzata, e $\sigma_{\simeq}$ la sua componente superficiale (t). 
Si ha dunque, prendendo la divergenza di [j] :

$\left(\Delta_{2} g\right)_{\Sigma}=\Delta, g-H \operatorname{grad} g \times v-\operatorname{grad}(\operatorname{grad} g \times v)<v=\Delta_{2} g-H \frac{d g}{d v}-\frac{d^{2} g}{d v^{2}}$

in cui $H=$ div $v=-\frac{2 \omega^{2}}{g}-\frac{1}{g} \frac{d g}{d n} \grave{e}$ la curvatura media di $\searrow((1)$ è la velocità angolare di rotazione della Terra).

$\mathrm{Si}$ osservi ora che, nel mentre si ha ovriamente, su $\mathrm{L}$,

$$
\frac{d \underline{g}}{d v}=\frac{d g}{d n}
$$

non altrettanto può dirsi per le derivate seconde; ed infatti

$$
\begin{aligned}
& \frac{d^{2} g}{d v^{2}}=\operatorname{grad}(\operatorname{grad} g \times v) \times v=\frac{d \operatorname{grad} g}{d P} v \cdot v+\underset{\Sigma}{K} \operatorname{grad} g \times v= \\
& =\frac{d \operatorname{grad} g}{d r} v \cdot v-\frac{d \operatorname{grad} g}{d P} n \cdot n
\end{aligned}
$$

perché $K \sigma \widetilde{\Sigma}=\sigma_{\Sigma} ;$ invece

$$
\begin{array}{r}
\frac{d^{\circ} g}{d n^{2}}=\operatorname{grad}(\operatorname{grad} g \times n) \times n=\frac{d \operatorname{grad} g}{d P} n \cdot n+K \sigma \operatorname{grad} g \times n= \\
=\frac{d^{2} g}{d v^{2}}+\operatorname{grad} g \times \sigma n=\frac{d^{2} g}{d v^{2}}+g F^{2} \quad[10]
\end{array}
$$

in cui $F=\bmod \operatorname{grad}_{\Sigma} \lg _{\Sigma} g$ è la prima curvatura della linea di forza.

A sua volta, il calcolo di $\Delta . g$ fornisce $\left(^{4}\right)$

$$
\Delta_{2} g=g\left(C+F^{2}\right)
$$

dove

$$
C=H^{2}-2 K
$$

è la curvatura di Casorati di $\Sigma$ ( $K$ ne è la curvatura totale o gaussiana).

Tenendo conto di ciò, al $(\Delta, g)_{\Sigma}$ puó essere data una delle seguenti forme

$$
\begin{gathered}
\left(\Delta_{2} g\right)_{\mathbf{S}}=2 g\left(H^{*}-K+F^{2}\right)+2 \omega^{2} H-\frac{d^{2} g}{d n^{2}}= \\
=g\left(C+2 F^{2}+\frac{d H}{d n}\right)
\end{gathered}
$$

La prima in particolare ci dice che il $\left(\Delta_{\Sigma} g\right)_{\Sigma}$ differisce dalla derivata seconda della gravità secondo la verticale, per termini che dipendono dalla curvatura della superficie equipotenziale e della linea 
di forza, e che in generale non sono affatto trascurabili. Così ad esempio si avrebhe, per il campo normale della gravità (campo di Somigliana) alla latitudine di $45^{\circ}$ :

$$
\frac{d^{*} g}{d n^{2}}=-14,495 \times 10^{-15} \mathrm{~cm}^{-1} \mathrm{sec}^{-2},
$$

nel mentre i termini derivanti dalla curvatura del campo ammontano a $14,501 \times 10^{5} \mathrm{~cm}^{-1}$ sec $*$, tanto che il $\Delta$, superficiale della gravità risulta uguale a $0,006 \times 10^{-5} \mathrm{~cm}^{-1} \mathrm{sec}^{-2}$.

5. Altre formule espressive, che possono tornare a volte utili, si hanno quando si consideri invece del campo $g$ della gravità, quello del suo logaritmo naturale.

La considerazione del logaritmo della gravita, anziché della gravità stessa, presenta il vantaggio di attribuire al suo gradiente le dimensioni di una curvatura; ed alle sue derivate seconde (od alla divergenza del suo gradiente) le dimensioni di una curvatura totale. Ciò i mostrato chiaramente dalle formule che seguono:

$$
\operatorname{grad} \lg g=F N^{\prime}-\left(\frac{2 \omega^{2}}{g}+H\right)^{n} \text { (formula di Bruns) }
$$

in cui $N^{\prime}$ è il versore della normale principale alla linea di forza.

Facili calcoli danno inoltre

$$
\Delta_{2} \lg g=-2 K+\frac{4 \omega^{4}}{g^{2}}+\frac{4 \omega^{2}}{g} \frac{d \lg g}{d n}=C-\left(\frac{d \lg g}{d n}\right)^{2}
$$

e poi

$$
\left(\Delta_{0} \lg g\right)_{\triangle}=C+F^{2}+\frac{d H}{d n}
$$

Trieste - Istituto di Topografia e Geodesia dellUniv. - Marzo 1953.

\section{RIASSUNTO}

Si richiamano alcune ricerche di Pizzetti, che consentono di ottenere, con procedimenti applicalili in pratica, la divergenza del gradiente superficiale della gravità (secondo parametro differenziale di Beltrami della gravità in superficie). Queste ricerche vengono messe 
in relazione con $i$ metodi della riduzione regionale e delle derivate seconde, che sono stati proposti recentemente dagli Autori americani per le pratiche applicazioni in gravimetria.

Nel caso in cui la superficie sulla quale si calcola tale divergenza sia di livello, il secondo parametro differenziale si esprime assai semplicemente mediante gli elementi geometrici e meccanici del campo.

\section{$S U M M A R Y$}

Some formulae given by Pizzelti are recalled, which allow to obtain by practical procedures the divergence of the surface gradient of gravity (second differential parameter of Beltrami of surface gravity). Such researches are related to the methods of regional reduction and of the second derivatives, which have been recently proposed for practical applications by American authors.

Whenever the surface considered be a level surface of the potential field, the second differential parameter may be easily expressed by means of the geometric and dynamic elements of the field itself.

\section{BIBLIOGR AFIA}

(1) W. Raymond Griffix, Residual Gravity in Theory and Practice. Geophysics, vol. XIV, January, 1949. - Thomas A. Elkins, The Second Derivaives Method of Gravity Interpretation. Geophysics, vol. XVI, January, 1951. - J. J. JАкоSкY, Exploration Geophysics, pag. 415 e segg., Los Angeles, 1950.

(2) P. Pizzettr, Sul significato geometrico del secondo parametro differenziale di una funzione sopra una superficie qualunque. Rend. R. Acc. dei Lincei, vol. XVIII, $1^{\circ}$ sem., 1909.

(3) C. Bunni-Fonti e R. Mancolongo, Analisi V'ettoriale Generale e Applica, zioni. Trasformazioni lineari, Bologna, Zanichelli, 1929, pag. 227.

(4) A. Manussi, Fondamenti di Geodesia intrinseca. Memoria n. 7 della Commissione Geodetica Italiana, Milano, 1950. 